\title{
Os aspectos morfológicos do Carcinoma epidermóide oral e sua correlação com parâmetros clínicos e prognóstico dos pacientes afetados
}

\section{Rafael Cabral de Albuquerque Souza ${ }^{1}$; Tarsila de Carvalho Freitas Ramos ${ }^{2}$, Marla Smille$^{3}$; Aline Moreira ${ }^{4}$;Gabriel Levi da Silva Cunha ${ }^{5}$ \\ 1.Bolsista PROBIC/UEFS, Graduando em odontologia, Universidade Estadual de Feira de Santana, email: rafael_cabral27@live.com \\ 2.Orientador, Departamento de Saúde, Universidade Estadual de Feira de Santana, email: tarsilafreitas@yahoo.com.br \\ 3.Pesquisadora do Núcleo de Câncer Oral, Departamento de Saúde, Universidade Estadual de Feira de Santana, email: m.smille@hotmail.com \\ 4. Participante do projeto, Graduando em odontologia, Universidade Estadual de Feira de Santana, email:alineemoreia94@gmail.com \\ 5. Participante do projeto, Graduando em odontologia, Universidade Estadual de Feira de Santana, email: gabriellscunha@gmail.com}

\section{Introdução}

Palavras-chave: Câncer bucal; Estomatologia; Patologia bucal

O câncer bucal é o sexto câncer mais comum do mundo e a incidência de novos casos indicam um continuo crescimento nos países desenvolvidos. Dentre as neoplasias bucais, o carcinoma de células escamosas (CCE) é o mais comum, representando mais de $90 \%$ dessas neoplasias. Embora a ocorrência de câncer da cavidade oral seja maior nos países orientais, o Brasil possui uma das maiores taxas de câncer de boca do mundo (MARTÍNEZ et al., 2011; MONTEIRO et al., 2014). O estadiamento clínico TNM (sistema de estadiamento clínico mais utilizado que avalia o tamanho do tumor e a extensão da disseminação metastática) vem sendo a forma mais comumente utilizada na avaliação dos tumores. Entretanto, este método tem sido considerado falho para a determinação de prognóstico em $\mathrm{CCE}$ de boca. A utilização de características histopatológicas, para esta mesma função, tem sido amplamente estudada e resultados mais satisfatórios foram obtidos (ALVES et al., 2011; MARTÍNEZ et al., 2011). Portanto, o conhecimento da patologia (macro e microscópica), com suas diversas apresentações clínicas e suas consequentes implicações no curso da doença é essencial para os profissionais de saúde que se dedicam à prevenção, deteç̧ão precoce e tratamento do câncer de cabeça e pescoço, em especial do carcinoma epidermóide de boca, o tipo histológico mais prevalente na cavidade oral (LOURENÇO et al., 2007; ALMEIDA et al., 2011).

\section{Metodologia}

Foi realizado um estudo retrospectivo incluindo a revisão de prontuários clínicos odontológicos da Disciplina Estudos Integrados XIV da UEFS e prontuários médicos da Unidade de Alta Complexidade em Oncologia (UNACON) do Hospital Dom Pedro em Feira de Santana-Ba, Brasil, visando à busca de informações clínicas e sócio- 
demográficas dos pacientes afetados pelo Carcinoma Epidermóide oral, além de informações sobre o Estadiamento Clínico (Sistema TNM) e terapêutica realizada. Realizou-se, ainda, uma revisão retrospectiva das lâminas histológicas com diagnóstico de Carcinoma Epidermóide Oral nos arquivos do Laboratório de Patologia Bucal da UEFS, CION-UEFS. A gradação histológica de malignidade foi realizada na área invasiva do tumor por dois patologistas em secções histológicas de $3 \mu \mathrm{m}$ de espessura coradas pela hematoxilina e eosina. Para essa gradação, os espécimes foram avaliados e classificados através do sistema de gradação histológica de malignidade desenvolvido por Bryne (1998). Os resultados obtidos foram submetidos às análises estatísticas com testes não-paramétricos (Tukey; Pearson; Qui2). O estudo foi realizado observando a Resolução 196/96 (Cap. IX.2) que diz respeito à pesquisa envolvendo seres humanos (BRASIL, 1997). Os prontuários dos pacientes investigados continham o TCLE, onde era esclarecido que todas as informações contidas nos mesmos poderiam ser utilizadas em pesquisas e divulgações científicas. Vale ressaltar que este estudo se encontra registrado no Comitê de Ética da Universidade Estadual de Feira de Santana (CEPUEFS) sob protocolo, No 015/2008, CAAE 0015.0.059.000-08.

\section{Resultados e discussão}

Foram selecionados 14 prontuários baseados nos critérios de inclusão e exclusão desta pesquisa. A maioria dos pacientes eram do gênero masculino representando $42,9 \%$ da amostra $(n=8)$ e $38,5 \%$ eram melanodermas. Dos pacientes afetados, 69,2\% $(n=9)$ relataram hábito tabagista e 61,5\% ingestão de bebidas alcoólicas. (Tabela 1). Em relação aos aspectos histológicos, 64,3\% $(n=9)$ dos tumores analisados exibiam arranjo histológico misto (ninhos e cordões); 92,9\% ( $\mathrm{n}=13)$ tinham infiltrado inflamatório moderado e 71,4\% $(n=10)$ eram moderadamente diferenciados (Tabela 2). Em relação ao estadiamento clinico, 3 pacientes (100\%) foram estadiados em Graus I e II, sendo os mesmos submetidos à cirurgia como modalidade terapêutica exclusiva, já os pacientes estadiados em Graus III e IV, representados por 5 pacientes (100\%) desta amostra, foram submetidos à terapia conjugada com quimioterapia e radioterapia (Tabela 3 ). Quando aplicado o teste Qui Quadrado de Person não foi encontrado associação estatisticamente significante para as variáveis Localização da lesão x Estadiamento, Hábito de fumar x Estadiamento, Gradação Histológica x Estadiamento; porém Estadiamento $\mathrm{x}$ Tratamento, encontrou-se $\mathrm{p}=0,035$. A explicação possível advém do 
fato do pequeno número na amostra. A média de idade foi de 57 anos, com desviopadrão de 15 anos. Em estudo realizado por Santos et al. (2012), os pacientes acometidos por carcinoma epidermóide apresentavam entre 51 e 60 anos, demonstrando, assim como em nosso estudo, que pacientes acima dos 40 anos estão numa faixa de risco para o desenvolvimento de câncer oral. O gênero masculino foi o mais acometido pelo carcinoma epidermóide na amostra pesquisada, corrobando com o estudo de Francio et al, 2011, onde 78,42\% da amostra com diagnóstico de carcinoma escamocelular oral eram homens. O gênero masculino, portanto, pode ser um fator de risco para desenvolvimento deste tipo de Câncer Oral. A maioria dos pacientes com o diagnóstico de carcinoma epidermóide, nesse estudo, possuíam o hábito de fumar e consumir bebidas alcoólicas, o que está em consonância com o estudo de Volkweis et al, 2014, realizado em um Centro de Especialidades Odontológicas na cidade de Porto Alegre, onde $61,64 \%$ dos pacientes eram tabagistas. Sabe-se que o fumo se constitui no principal fator de risco para o desenvolvimento dos carcinomas, devido ao seu potencial carcinogênico. O álcool atua nesse processo numa relação sinérgica ao tabaco, potencializando o desenvolvimento do câncer (SANTOS et al, 2010). Neste estudo constatou-se que o tratamento nos pacientes Grau III e IV tiveram a quimioterapia e radioterapia como tratamento de escolha, isso mostra que em graus avançados a cirurgia não terá uma resolutividade, por mutilar o paciente sem ter uma resolutividade.

Tabela 1. Características sociodemográficas dos pacientes com diagnóstico de carcinoma escamocelular,

\begin{tabular}{|c|c|c|}
\hline CARACTERÍSTICAS & $n$ & $\%$ \\
\hline \multicolumn{3}{|l|}{ Gênero } \\
\hline Masculino & 8 & $57.1 \%$ \\
\hline Feminino & 6 & $42,9 \%$ \\
\hline \multicolumn{3}{|l|}{ Cor da pele* } \\
\hline Pardo & 2 & $15,4 \%$ \\
\hline Leucoderma & 4 & $30,8 \%$ \\
\hline Melanoderma & 5 & $38,5 \%$ \\
\hline Xantoderma & 2 & $15,4 \%$ \\
\hline \multicolumn{3}{|l|}{ Hábiro de fumar* } \\
\hline sim & 9 & $69,2 \%$ \\
\hline Nào & 4 & $30,8 \%$ \\
\hline \multicolumn{3}{|l|}{ Consumo de álcool* } \\
\hline $\operatorname{sim}$ & 8 & $61,5 \%$ \\
\hline Não & 5 & $38,5 \%$ \\
\hline
\end{tabular}


Tabela 2. Características histológicas dos pacientes com diagnóstico de carcinoma escamocelular,

\begin{tabular}{lcc} 
& CRLB/NUCAO, Feira de Santana Bahia, 2017. \\
\hline Características histológicas & $\mathbf{N}$ & $\%$ \\
\hline Arranjo Histológico & 1 & $7,1 \%$ \\
\hline Cordöes epiteliais & 3 & $21,4 \%$ \\
\hline Ninhos epiteliais & 9 & $7,3 \%$ \\
\hline Ambos arranjos & 1 & $7,1 \%$ \\
\hline Nenhum dos dois & & $7,1 \%$ \\
\hline Intensidade do infiltrado inflamatório & 1 & $92,9 \%$ \\
\hline Moderado & 13 & $21,4 \%$ \\
\hline Intenso & & $71,4 \%$ \\
\hline Grau de diferenciação do tumor & 3 & $7,1 \%$ \\
\hline Bem diferenciado & 10 & \\
\hline Moderadamente diferenciado & 1 & \\
\hline Pobremente diferenciado & & \\
\hline
\end{tabular}

Tabela 3. Associação entre Tipo de tratamento e estadiamento em individuos com carcinoma escamocuelar, UNACOM, Feira de Santana Bahia, 2017.

\begin{tabular}{|c|c|c|c|c|c|}
\hline \multirow[t]{2}{*}{ Tipo de tratamento } & \multicolumn{5}{|c|}{ Estadiamento } \\
\hline & Graus III e IV & $\%$ & Graus I e II & $\%$ & $\mathbf{p}^{*}$ \\
\hline Radioterapia & 1 & 100 & O & O & \\
\hline Quimioterapia & 1 & 100 & o & O & \\
\hline $\begin{array}{l}\text { Radioterapia e } \\
\text { quimioterapia }\end{array}$ & 5 & 100 & $\mathbf{O}$ & $\mathbf{O}$ & 0,035 \\
\hline Cirurgia & O & o & 3 & 100 & \\
\hline $\begin{array}{l}\text { Cirurgia, quimioterapia } \\
\text { e radioterapia }\end{array}$ & 1 & 100 & $\mathbf{O}$ & o & \\
\hline Cirurgia e radioterapia & 1 & 100 & $\mathbf{O}$ & o & \\
\hline
\end{tabular}

* Teste do qui-quadrado

\section{Conclusão}

Os pacientes portadores de Carcinoma Epidermóide diagnosticado no CRLB eram em sua maioria do gênero masculino, melanoderma e tabagistas; os tumores, morfologicamente, eram em sua maioria moderadamente diferenciados e com arranjo em ninhos e cordões epiteliais; a maioria dos tumores foram estadiados clinicamente em Graus III e IV, demonstrando a busca tardia dos pacientes pelo tratamento; quanto maior o estadiamento clínico dos tumores mais complexo o tratamento selecionado.

\section{Referências}

ALMEIDA, F. C. S; CAZAL, C; NUNES, F. D; ARAÚJO, M. E; DIAS, R.B; SILVA, D. P. Fatores prognósticos no câncer de boca. Rev. Brasileira de Ciências da Saúde. V. 15, n.4, p 471-478, 2011.

ALVES, C. C. M; NETTO, F. O. G; SOUSA, S. F; BERNARDES, V. F; AGUIAR, M. C. F. Carcinoma de células escamosas de boca: Relação entre graduação histopatológica e características clínicas da neoplasia. Rev. Pesquisa Brasileira em Odontopediatria e Clínica Integrada. V.11, n.5, p 485$489,2011$.

LOURENÇO, S. M. Q. C; SCHUELER, A. F; CAMISASCA, D. R; LINDENBLATT, R. C; BERNADO, V. G. Classificações histopatológicas para carcinoma de células escamosas da cavidade oral: Revisão de sistemas propostos. Rev. Brasileira de Cancerologia. V.53, n. 3, p 325-333, 2007.

MARTÍNEZ, E. A; URIBE, A. P; FERNÁNDEZ, S. M; GÓMEZ, R. J; LÓPEZ, A. P. Características clínicas e histopatológicas del carcinoma escamocellular bucal em elperiodo 1990-2004 en Medellín, Colombia. Rev. Cubana de Estomatología. V.48, n.4, p 320-329, 2011.

MONTEIRO, L. S; AMARAL, J. B; VIZCAÍNO, J. R; LOPES, C. A; TORRES, F. O. A clinical-pathological and survival study of oral squamous cell carcinomas from a population of the north of Portugal. Medicina Oral, Patología Oral y Cirugía Bucal. V.19, n.2, p 120-126, 2014.

SANTOS, G. L; FREITAS, V. S; ANDRADE, M. C; OLIVEIRA, M. C. Fumo e álcool como fatores de risco para o câncer bucal. Odontol. Clin. Cient., v. 9, n. 2, p. 131-133, 2010. 
